\title{
CALIBRATION OF THE RADIOCARBON TIME SCALE FOR THE SOUTHERN HEMISPHERE: AD 1850-950
}

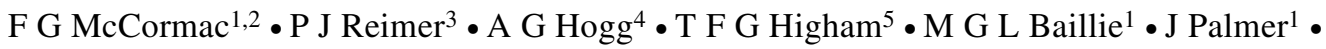 \\ M Stuiver 6
}

\begin{abstract}
We have conducted a series of radiocarbon measurements on decadal samples of dendrochronologically dated wood from both hemispheres, spanning 1000 years (McCormac et al. 1998; Hogg et al. this issue). Using the data presented in Hogg et al., we show that during the period AD $950-1850$ the ${ }^{14} \mathrm{C}$ offset between the hemispheres is not constant, but varies periodically ( $\sim 130$ yr periodicity) with amplitudes varying between 1 and $10 \%$ (i.e. $8-80 \mathrm{yr}$ ), with a consequent effect on the ${ }^{14} \mathrm{C}$ calibration of material from the Southern Hemisphere. A large increase in the offset occurs between AD 1245 and 1355. In this paper, we present a Southern Hemisphere high-precision calibration data set (SHCal02) that comprises measurements from New Zealand, Chile, and South Africa. This data, and a new value of $41 \pm 14 \mathrm{yr}$ for correction of the IntCal98 data for the period outside the range given here, is proposed for use in calibrating Southern Hemisphere ${ }^{14} \mathrm{C}$ dates.
\end{abstract}

\section{INTRODUCTION}

Regional radiocarbon offsets have been recognized in the past. Lerman et al. (1970), using wood from about AD 1835, showed that trees from the Southern Hemisphere dated older by approximately $30\left({ }^{14} \mathrm{C}\right)$ yr compared to identically aged Northern Hemisphere trees. More recently, Vogel et al. (1993) measured a 40-yr offset between hemispheres comparing data from the Netherlands and South Africa (wood spanning the yr AD 1835-1890). McCormac et al. (1998) measured hemispheric differences of $27 \mathrm{yr}$ (British Isles/New Zealand; 18th to 19th century) and Stuiver and Braziunas (1998) measured differences of $23 \mathrm{yr}$ (Western US/Chile; 19th century). Accepted theory attributes the difference to the influence of the larger expanse of ocean in the Southern Hemisphere and the atmosphere-ocean $\mathrm{CO}_{2}$ exchange. Thus, ${ }^{14} \mathrm{C}$ is not uniformly distributed throughout the troposphere but exhibits hemispheric differences as observed in terrigenous organic material. The long-term question has been, do these differences vary temporally?

Small differences in the dates of wood from different locations are extremely difficult to detect. If wood from different regions is measured in different laboratories, then small systematic differences between laboratories can easily mask any signal that may exist (McCormac et al. 1995). For this reason, we have measured replicates of the oak (Quercus petraea), cedar (Libocedrus bidwillii), and silver pine (Lagarostrobos colensoi) at the Queen's University of Belfast and The University of Waikato, New Zealand ${ }^{14} \mathrm{C}$ dating laboratories Hogg et al. (this issue). Weighted means of the differences in the wood measurements show consistent ${ }^{14} \mathrm{C}$ depletion or older ${ }^{14} \mathrm{C}$ ages in the Southern Hemisphere over the period AD 950-1850. After these dates, i.e. in the period AD 1895-1935, the results from both laboratories show a reversal such that the Northern Hemisphere is slightly depleted. This reversal has been previously attributed to the burning of fossil fuel in the industrialized Northern Hemisphere (McCormac et al. 1998; Stuiver and Braziunas 1998).

In Figure 1, the mean differences of the data from Hogg et al. (SH offset) are plotted along with a 3point moving average. A $120-130 \mathrm{yr}$ periodicity with varying amplitude is clearly visible in the 3-

\footnotetext{
${ }^{1}$ School of Archaeology and Palaeoecology, The Queen's University of Belfast, Belfast BT7 1NN, Northern Ireland. ${ }^{2}$ Corresponding author. Email: f.mccormac@qub.ac.uk.

${ }^{3}$ Center for Accelerator Mass Spectrometry L-397, Lawrence Livermore National Laboratory, P.O. Box 808, Livermore, California 94550, USA.

${ }^{4}$ Radiocarbon Dating Laboratory, University of Waikato, Private Bag 3105, Hamilton, New Zealand. ${ }^{5}$ Oxford Radiocarbon Accelerator Unit, Oxford University, 6 Keble Rd, Oxford, OX1 3QJ, England.

${ }^{6}$ Quaternary Isotope Laboratory, University of Washington, Seattle, Washington 98195-1360, USA.
} 
point moving average. This periodicity is well above the red noise $95 \%$ confidence limit in the Fourier spectral analysis (Mitchell et al. 1966) (Figure 2) and was confirmed as one of the principle components of variation in the NS offset using singular spectrum analysis (Dettinger et al. 1995; Vautard et al. 1992). Spectral analysis was carried out on the data excluding the period before AD 1400 and the 120-130 yr periodicity persisted. A large increase in the SH offset is seen between AD 1245 and 1355.

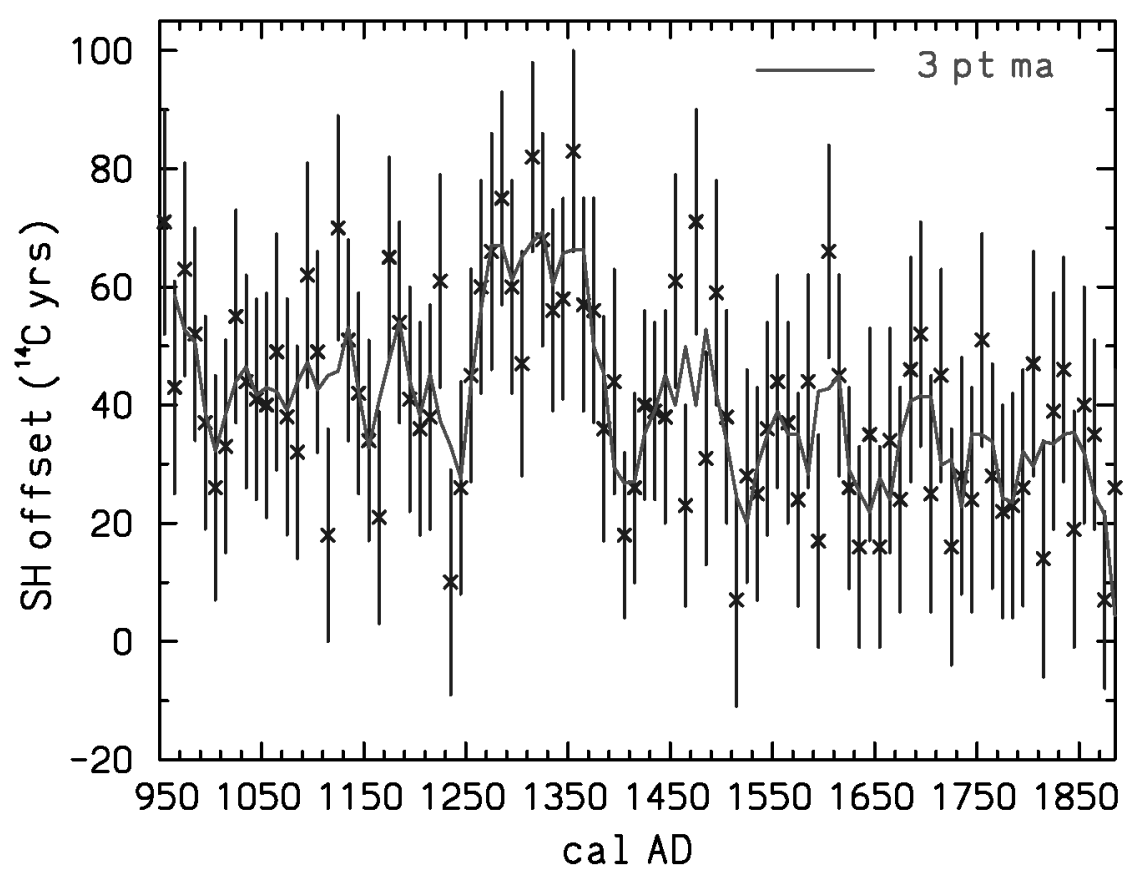

Figure 1 Difference between combined Southern and Northern Hemisphere measurements with a 3 -point moving average superimposed

Previous work to establish the Southern Hemispheric offset has produced several high-precision datasets (Vogel et al. 1993; Sparks et al. 1995; Stuiver and Braziunas 1998). Although some of these datasets are based on ring-counted sequences rather than precise dendrochronological dates, we compared these results from the high-precision laboratories at the Universities of Washington (QL), Pretoria and Groningen (Pta/Grn), and the Rafter Radiocarbon Laboratory (NZ) with the combined measurements from Belfast and Waikato. The University of Washington data from Chile (previously published in graphical form in Stuiver and Braziunas 1998) is given in Table 1. The other data has been published previously. The offsets between the laboratories are shown in Table 2. There is a high level of agreement between our dataset and the QL and Pta/Grn measurements in terms of both the mean offset values and the spread of measurements. The mean offset value with the NZ measurements is larger especially for the period from AD 1335-1445. The NZ measurements were therefore not included in the Southern Hemisphere calibration dataset.

The combined Southern Hemisphere data are given in Table 3. Data have been combined by the method used for IntCal98 and described in Stuiver et al. (1998). The error multiplier $(k)$ is a convenient measure of the degree to which the errors in measurement have been accounted for (Stuiver and Pearson 1986) with a value of 1.0 indicating that all sources of error in the measurements have 


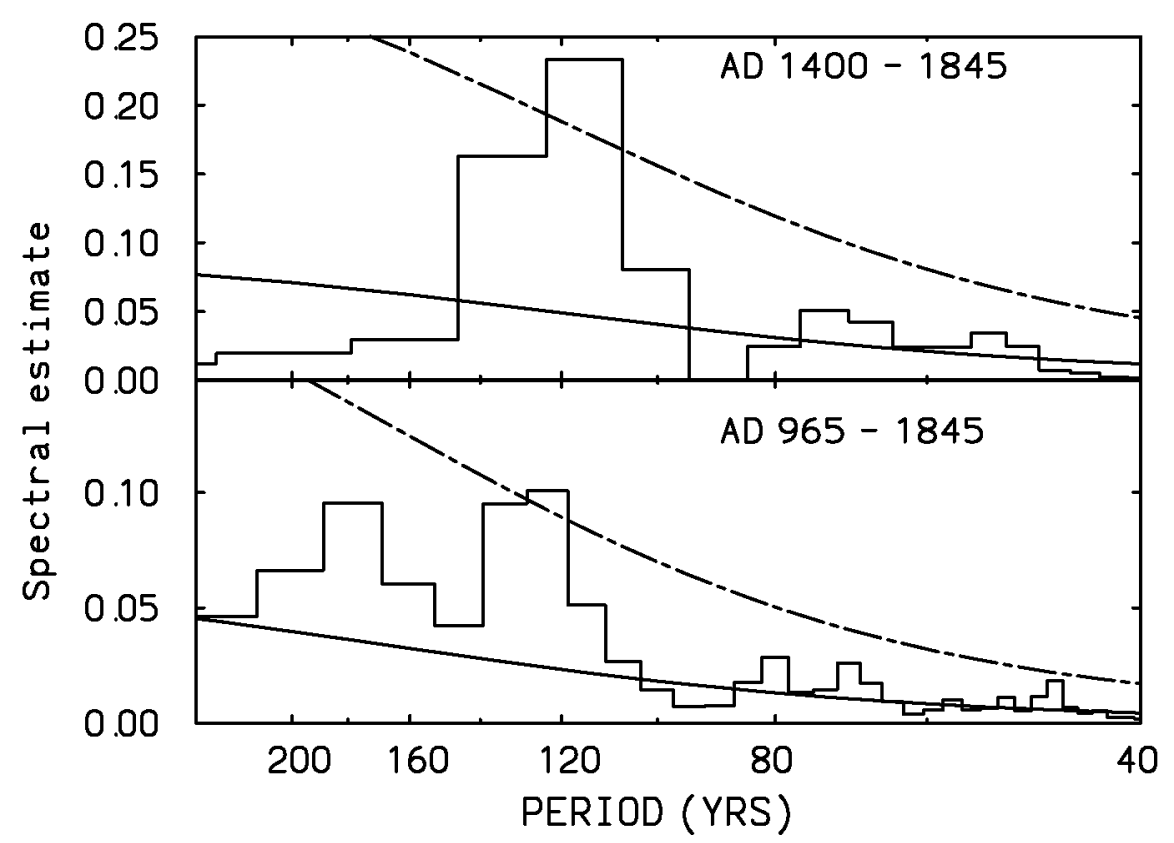

Figure 2 Spectral analysis of the difference between Northern and Southern Hemisphere measurements. The difference was detrended using a 20-point moving average and smoothed with a 3-point moving average. The $95 \%$ red noise confidence level is indicated by the dashed line.

been included, whereas values below 1.0 indicate an overestimate of the error. An error multiplier of 1.2 was used for the combined data from 1665 to 1955 based on the largest $k$ value obtained for the differences between labs (Table 2). No error multiplier was applied to the data prior to 1665 as the $k$ values indicate overestimates of the uncertainty were included in the Belfast and Waikato datasets. Taking the SH offset determined by subtracting the data in Table 3 from IntCal98 (Figure 3 ), we obtain a mean offset of $41 \pm 1 \mathrm{yr}$ with a standard deviation based on the spread in the differences of \pm 14 . This value is similar to that found by Vogel et al. (1993), but encompasses a much longer time span. The offset may be used as a correction for Southern Hemisphere ${ }^{14} \mathrm{C}$ measurements beyond the range of the new data; however, past changes in the ocean-atmosphere regime could and will almost certainly have altered the inter-hemispheric difference in ${ }^{14} \mathrm{C}$ reservoirs especially beyond the Holocene. We therefore would suggest using this offset for Holocene samples only and recommend the use of the standard deviation of \pm 14 to encompass the time variation we observe over the last millennium.

\section{DISCUSSION}

The ocean effect on atmospheric ${ }^{14} \mathrm{C}$ gradients has previously been modelled (Stuiver and Braziunas 1998; Levin et al. 1987; Braziunas et al. 1995). The relative depletion of ${ }^{14} \mathrm{C}$ in the Southern Hemisphere atmosphere before 1885 can be attributed to the differences in ocean circulation and $\mathrm{CO}_{2}$ exchange between ocean and atmosphere in the 2 hemispheres. In the Southern Ocean, surface ocean ${ }^{14} \mathrm{C}$ is low, reflecting the exposure of old, deep waters at the surface. While there is a similar but smaller depletion of ${ }^{14} \mathrm{C}$ in the North Pacific, the ${ }^{14} \mathrm{C}$ in North Atlantic surface waters remains high reflecting their origins in low latitudes and longer exposure time to the atmosphere. We used a 2-hemisphere ocean-atmosphere box diffusion model with our Northern Hemispheric ${ }^{14} \mathrm{C}$ record as 
input to identify possible causes of the increased SH offset for this period. We found that, by using the original parameters of Stuiver and Braziunas (1998), the model Southern Hemisphere atmosphere fit the data exceeding well from $\mathrm{AD} 1400$ to 1840 , but was less depleted than the ${ }^{14} \mathrm{C}$ values of the preceding period. Increases in the air-sea $\mathrm{CO}_{2}$ exchange rate and the eddy diffusivity did not significantly alter the model output. Addition of an advection to the deep ocean and the subsequent replacement by upwelling in the Southern Hemisphere or an increase in the tropospheric exchange rate for the period AD 1200-1400 improved the fit considerably. This modelling work and discussion of the periodicity in the SH offset is expanded in the forthcoming paper by Reimer et al.

Table 1 Measurements on Chilean wood samples (Notofagus dombeyi) at the University of Washington. Uncertainties are based on counting statistics. ${ }^{14} \mathrm{C}$ ages are not given for samples later than AD 1958, after which ${ }^{14} \mathrm{C}$ from nuclear testing makes ${ }^{14} \mathrm{C}$ ages meaningless.

\begin{tabular}{lccccrccc}
\hline $\begin{array}{c}\text { Year AD } \\
\text { (midpoint) }\end{array}$ & $\begin{array}{c}\text { Number } \\
\text { of rings }\end{array}$ & \multicolumn{2}{c}{${ }^{14} \mathrm{C}(\mathrm{BP})$} & \multicolumn{1}{c}{$\Delta^{14} \mathrm{C}(\% \circ)$} & & $\begin{array}{c}\delta^{13} \mathrm{C} \\
(\% o)\end{array}$ \\
\hline 1665 & 10 & 271 & \pm & 17 & 0.8 & \pm & 2.1 & -24.6 \\
1671.5 & 3 & 205 & \pm & 20 & 8.3 & \pm & 2.5 & -24.0 \\
1674.5 & 3 & 219 & \pm & 13 & 6.1 & \pm & 1.7 & -24.0 \\
1677 & 2 & 200 & \pm & 10 & 8.2 & \pm & 1.3 & -23.9 \\
1679 & 2 & 192 & \pm & 13 & 9.0 & \pm & 1.6 & -23.6 \\
1681 & 2 & 178 & \pm & 13 & 10.5 & \pm & 1.7 & -23.4 \\
1683 & 2 & 177 & \pm & 13 & 10.4 & \pm & 1.6 & -23.3 \\
1685 & 2 & 171 & \pm & 15 & 10.8 & \pm & 1.9 & -23.2 \\
1687 & 2 & 196 & \pm & 13 & 7.5 & \pm & 1.6 & -23.9 \\
1688.5 & 5 & 163 & \pm & 15 & 11.5 & \pm & 1.9 & -25.0 \\
1689 & 2 & 172 & \pm & 15 & 10.3 & \pm & 1.9 & -24.1 \\
1691 & 2 & 165 & \pm & 15 & 10.9 & \pm & 1.9 & -23.7 \\
1692.5 & 1 & 173 & \pm & 20 & 9.8 & \pm & 2.6 & -23.7 \\
1693.5 & 1 & 185 & \pm & 10 & 8.1 & \pm & 1.2 & -23.4 \\
1694.5 & 1 & 135 & \pm & 17 & 14.2 & \pm & 2.1 & -23.0 \\
1695.5 & 1 & 188 & \pm & 14 & 7.4 & \pm & 1.8 & -23.3 \\
1696.5 & 1 & 159 & \pm & 9 & 11.0 & \pm & 1.2 & -23.5 \\
1697.5 & 1 & 161 & \pm & 13 & 10.7 & \pm & 1.6 & -23.3 \\
1698.5 & 1 & 177 & \pm & 13 & 8.5 & \pm & 1.6 & -23.2 \\
1698.5 & 5 & 154 & \pm & 10 & 11.3 & \pm & 1.3 & -24.4 \\
1699.5 & 1 & 156 & \pm & 14 & 11.0 & \pm & 1.8 & -23.3 \\
1700.5 & 1 & 149 & \pm & 9 & 11.8 & \pm & 1.2 & -23.4 \\
1701.5 & 1 & 154 & \pm & 13 & 11.1 & \pm & 1.6 & -23.4 \\
1702.5 & 1 & 151 & \pm & 14 & 11.2 & \pm & 1.8 & -23.2 \\
1703.5 & 1 & 152 & \pm & 13 & 11.0 & \pm & 1.6 & -22.8 \\
1703.5 & 5 & 141 & \pm & 11 & 12.5 & \pm & 1.4 & -24.2 \\
1705 & 2 & 167 & \pm & 9 & 8.9 & \pm & 1.1 & -23.0 \\
1706.5 & 1 & 138 & \pm & 9 & 12.4 & \pm & 1.1 & -22.9 \\
1707.5 & 1 & 149 & \pm & 13 & 10.9 & \pm & 1.7 & -22.2 \\
1708.5 & 1 & 178 & \pm & 10 & 7.2 & \pm & 1.3 & -22.7 \\
1708.5 & 5 & 146 & \pm & 17 & 11.1 & \pm & 2.1 & -24.0 \\
1709.5 & 1 & 154 & \pm & 10 & 10.0 & \pm & 1.2 & -23.1 \\
& & & & & & & &
\end{tabular}


Table 1 Measurements on Chilean wood samples (Notofagus dombeyi) at the University of Washington. Uncertainties are based on counting statistics. ${ }^{14} \mathrm{C}$ ages are not given for samples later than AD 1958, after which ${ }^{14} \mathrm{C}$ from nuclear testing makes ${ }^{14} \mathrm{C}$ ages meaningless. (Continued)

\begin{tabular}{lccllrlll}
\hline $\begin{array}{l}\text { Year AD } \\
\text { (midpoint) }\end{array}$ & $\begin{array}{r}\text { Number } \\
\text { of rings }\end{array}$ & \multicolumn{2}{c}{${ }^{14} \mathrm{C}(\mathrm{BP})$} & \multicolumn{1}{c}{$\Delta^{14} \mathrm{C}(\%)$} & & \multicolumn{1}{c}{$(\%)$} \\
\hline 1710.5 & 1 & 145 & \pm & 13 & 11.1 & \pm & 1.6 & -22.8 \\
1711.5 & 1 & 144 & \pm & 15 & 11.1 & \pm & 1.8 & -22.8 \\
1712.5 & 1 & 140 & \pm & 13 & 11.4 & \pm & 1.6 & -22.8 \\
1713.5 & 1 & 149 & \pm & 15 & 10.1 & \pm & 1.8 & -23.0 \\
1713.5 & 5 & 149 & \pm & 15 & 10.2 & \pm & 1.9 & -24.3 \\
1714.5 & 1 & 136 & \pm & 13 & 11.7 & \pm & 1.6 & -22.9 \\
1715.5 & 1 & 127 & \pm & 7 & 12.7 & \pm & 0.9 & -23.1 \\
1716.5 & 1 & 144 & \pm & 8 & 10.5 & \pm & 1.1 & -23.0 \\
1717.5 & 1 & 137 & \pm & 14 & 11.2 & \pm & 1.7 & -23.1 \\
1718.5 & 1 & 145 & \pm & 14 & 10.0 & \pm & 1.7 & -22.7 \\
1719.5 & 1 & 134 & \pm & 14 & 11.3 & \pm & 1.8 & -22.3 \\
1720.5 & 1 & 155 & \pm & 14 & 8.5 & \pm & 1.7 & -21.6 \\
1723 & 4 & 169 & \pm & 14 & 6.5 & \pm & 1.7 & -22.3 \\
1727.5 & 5 & 169 & \pm & 11 & 6.0 & \pm & 1.4 & -22.8 \\
1732.5 & 5 & 203 & \pm & 15 & 1.1 & \pm & 1.8 & -22.0 \\
1737.5 & 5 & 202 & \pm & 14 & 0.6 & \pm & 1.7 & -21.7 \\
1742.5 & 5 & 215 & \pm & 14 & -1.7 & \pm & 1.7 & -22.0 \\
1747.5 & 5 & 215 & \pm & 14 & -2.1 & \pm & 1.8 & -21.8 \\
1752.5 & 5 & 236 & \pm & 14 & -5.4 & \pm & 1.7 & -21.9 \\
1757.5 & 5 & 190 & \pm & 14 & -0.3 & \pm & 1.8 & -22.0 \\
1762.5 & 5 & 213 & \pm & 14 & -3.7 & \pm & 1.7 & -22.3 \\
1767.5 & 5 & 235 & \pm & 14 & -7.1 & \pm & 1.7 & -21.8 \\
1772.5 & 5 & 243 & \pm & 14 & -8.7 & \pm & 1.7 & -22.0 \\
1773 & 5 & 206 & \pm & 12 & -4.1 & \pm & 1.5 & -22.0 \\
1776.5 & 3 & 207 & \pm & 10 & -4.7 & \pm & 1.3 & -21.5 \\
1779 & 2 & 212 & \pm & 9 & -5.6 & \pm & 1.1 & -21.7 \\
1781 & 2 & 224 & \pm & 14 & -7.4 & \pm & 1.7 & -21.5 \\
1783 & 2 & 223 & \pm & 10 & & \pm & & -21.6 \\
1785 & 2 & 208 & \pm & 15 & -5.9 & \pm & 1.8 & -21.2 \\
1787 & 2 & 242 & \pm & 12 & -10.3 & \pm & 1.4 & -20.9 \\
1789 & 2 & 226 & \pm & 15 & -8.6 & \pm & 1.8 & -21.1 \\
1791 & 2 & 253 & \pm & 14 & -12.2 & \pm & 1.8 & -21.1 \\
1793 & 2 & 267 & \pm & 15 & -14.1 & \pm & 1.8 & -21.3 \\
1795 & 2 & 224 & \pm & 10 & -9.1 & \pm & 1.2 & -21.3 \\
1797 & 2 & 240 & \pm & 15 & -11.3 & \pm & 1.8 & -20.8 \\
1799 & 2 & 198 & \pm & 11 & -6.3 & \pm & 1.4 & -20.6 \\
1801 & 2 & 193 & \pm & 10 & -6.0 & \pm & 1.2 & -21.1 \\
1803 & 2 & 165 & \pm & 15 & -2.7 & \pm & 1.8 & -21.5 \\
1804.5 & 1 & 170 & \pm & 14 & -3.5 & \pm & 1.7 & -21.5 \\
1805.5 & 1 & 173 & \pm & 14 & -4.0 & \pm & 1.8 & -21.3 \\
1807 & 2 & 185 & \pm & 14 & -5.7 & \pm & 1.7 & -21.6 \\
& & & & & & & &
\end{tabular}


Table 1 Measurements on Chilean wood samples (Notofagus dombeyi) at the University of Washington. Uncertainties are based on counting statistics. ${ }^{14} \mathrm{C}$ ages are not given for samples later than AD 1958, after which ${ }^{14} \mathrm{C}$ from nuclear testing makes ${ }^{14} \mathrm{C}$ ages meaningless. (Continued)

\begin{tabular}{lcrllrlll}
\hline $\begin{array}{l}\text { Year AD } \\
\text { (midpoint) }\end{array}$ & $\begin{array}{r}\text { Number } \\
\text { of rings }\end{array}$ & \multicolumn{2}{c}{${ }^{14} \mathrm{C}(\mathrm{BP})$} & \multicolumn{1}{c}{$\Delta^{14} \mathrm{C}(\% \circ)$} & & \multicolumn{1}{c}{$(\%)$} \\
\hline 1809 & 2 & 160 & \pm & 12 & -2.8 & \pm & 1.4 & -20.9 \\
1810.5 & 1 & 149 & \pm & 16 & -1.6 & \pm & 2.0 & -21.1 \\
1811.5 & 1 & 136 & \pm & 14 & -0.1 & \pm & 1.7 & -21.1 \\
1812.5 & 1 & 134 & \pm & 14 & 0.0 & \pm & 1.8 & -21.2 \\
1814 & 2 & 156 & \pm & 13 & -2.9 & \pm & 1.7 & -20.6 \\
1815.5 & 1 & 142 & \pm & 14 & -1.3 & \pm & 1.7 & -20.8 \\
1816.5 & 1 & 131 & \pm & 14 & -0.1 & \pm & 1.7 & -20.7 \\
1817.5 & 1 & 120 & \pm & 13 & 1.2 & \pm & 1.7 & -20.7 \\
1819.5 & 3 & 97 & \pm & 14 & 3.8 & \pm & 1.7 & -20.9 \\
1824 & 2 & 104 & \pm & 13 & 2.3 & \pm & 1.6 & -21.6 \\
1828 & 5 & 107 & \pm & 14 & 1.5 & \pm & 1.8 & -21.8 \\
1833 & 5 & 143 & \pm & 11 & -3.6 & \pm & 1.3 & -21.5 \\
1838 & 5 & 156 & \pm & 13 & -5.8 & \pm & 1.6 & -21.2 \\
1843 & 5 & 164 & \pm & 10 & -7.3 & \pm & 1.2 & -21.5 \\
1847.5 & 5 & 170 & \pm & 14 & -8.6 & \pm & 1.7 & -21.8 \\
1850.5 & 3 & 148 & \pm & 9 & -6.4 & \pm & 1.1 & -22.2 \\
1853.5 & 3 & 132 & \pm & 15 & -4.6 & \pm & 1.9 & -22.6 \\
1856.5 & 3 & 142 & \pm & 15 & -6.3 & \pm & 1.8 & -22.2 \\
1862.5 & 3 & 110 & \pm & 14 & -3.0 & \pm & 1.8 & -22.6 \\
1865.5 & 3 & 129 & \pm & 11 & -5.8 & \pm & 1.3 & -22.7 \\
1868.5 & 3 & 119 & \pm & 14 & -4.9 & \pm & 1.8 & -22.7 \\
1871.5 & 3 & 144 & \pm & 14 & -8.4 & \pm & 1.7 & -22.6 \\
1874.5 & 3 & 169 & \pm & 10 & -11.7 & \pm & 1.2 & -22.5 \\
1877.5 & 3 & 136 & \pm & 15 & -8.1 & \pm & 1.8 & -22.8 \\
1880.5 & 3 & 134 & \pm & 14 & -8.1 & \pm & 1.8 & -23.1 \\
1883.5 & 3 & 135 & \pm & 15 & -8.7 & \pm & 1.8 & -22.9 \\
1886.5 & 3 & 115 & \pm & 15 & -6.5 & \pm & 1.8 & -22.9 \\
1889.5 & 3 & 121 & \pm & 15 & -7.7 & \pm & 1.8 & -23.0 \\
1898 & 5 & 110 & \pm & 10 & -7.4 & \pm & 1.3 & -21.4 \\
1903 & 5 & 146 & \pm & 9 & -12.4 & \pm & 1.0 & -21.2 \\
1908 & 5 & 133 & \pm & 10 & -11.4 & \pm & 1.2 & -21.1 \\
1913 & 5 & 147 & \pm & 8 & -13.7 & \pm & 1.0 & -20.6 \\
1918 & 5 & 125 & \pm & 14 & -11.6 & \pm & 1.8 & -20.7 \\
1923 & 5 & 133 & \pm & 14 & -13.2 & \pm & 1.8 & -20.0 \\
1928 & 5 & 123 & \pm & 14 & -12.6 & \pm & 1.8 & -20.2 \\
1933 & 5 & 158 & \pm & 14 & -17.5 & \pm & 1.8 & -20.7 \\
1938 & 5 & 146 & \pm & 14 & -16.6 & \pm & 1.7 & -20.4 \\
1943 & 5 & 162 & \pm & 14 & -19.1 & \pm & 1.7 & -20.6 \\
1948 & 5 & 164 & \pm & 15 & -20.0 & \pm & 1.8 & -21.3 \\
1953 & 5 & 177 & \pm & 15 & -22.1 & \pm & 1.8 & -20.8 \\
1958 & 5 & 187 & \pm & 15 & -24.0 & \pm & 1.8 & -20.9 \\
& & & & & & & &
\end{tabular}


Table 1 Measurements on Chilean wood samples (Notofagus dombeyi) at the University of Washington. Uncertainties are based on counting statistics. ${ }^{14} \mathrm{C}$ ages are not given for samples later than AD 1958, after which ${ }^{14} \mathrm{C}$ from nuclear testing makes ${ }^{14} \mathrm{C}$ ages meaningless. (Continued)

\begin{tabular}{|c|c|c|c|c|c|c|c|}
\hline $\begin{array}{l}\text { Year AD } \\
\text { (midpoint) }\end{array}$ & $\begin{array}{l}\text { Number } \\
\text { of rings }\end{array}$ & \multicolumn{2}{|c|}{${ }^{14} \mathrm{C}(\mathrm{BP})$} & \multicolumn{3}{|c|}{$\Delta^{14} \mathrm{C}(\% \circ)$} & $\begin{array}{l}\delta^{13} \mathrm{C} \\
(\% o)\end{array}$ \\
\hline 1963 & 5 & - & - & 107.6 & \pm & 2.0 & -21.3 \\
\hline 1968 & 5 & - & - & 402.6 & \pm & 2.0 & -21.3 \\
\hline 1973 & 5 & - & - & 552.7 & \pm & 2.6 & -21.4 \\
\hline 1978 & 7 & - & - & 431.9 & \pm & 3.5 & -20.8 \\
\hline
\end{tabular}

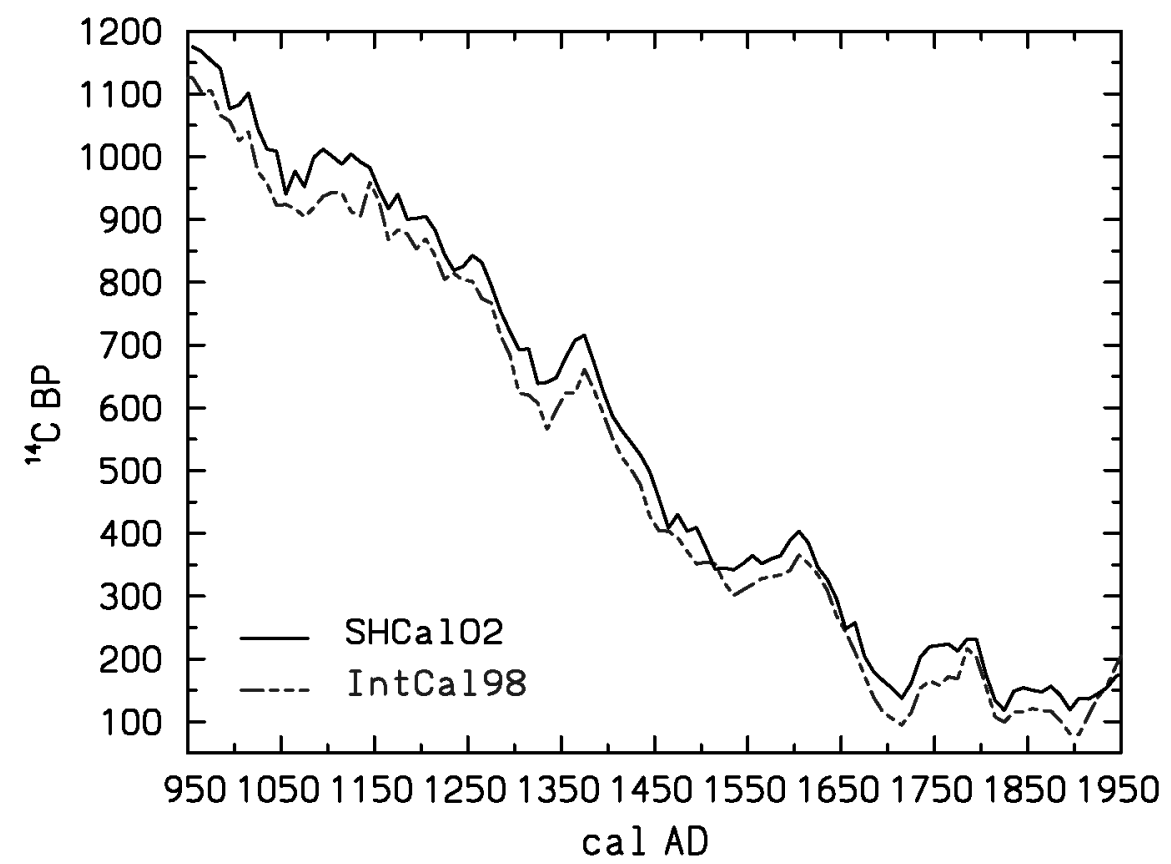

Figure 3 Comparison of the combined Southern Hemisphere datasets with IntCal

Table 2 Offsets between high-precision Southern Hemispheric datasets. $\sigma_{1}$ is the average standard deviation based on quoted laboratory errors and $\sigma_{2}$ is the observed standard deviation in the age difference. The error multiplier $\mathrm{k}=\sigma_{2} / \sigma_{1}$.

\begin{tabular}{lrcccrc}
\hline Laboratories & \multicolumn{1}{c}{ Offset } & \multicolumn{1}{c}{$\mathrm{s}_{1}$} & \multicolumn{1}{c}{$\mathrm{s}_{2}$} & $\mathrm{k}$ & \multicolumn{1}{c}{$\mathrm{N}$} & \multicolumn{1}{c}{ Interval } \\
\hline UB - Wk & $-3.9 \pm 2.5$ & 25.3 & 23.6 & 0.9 & 100 & AD 955-1945 \\
UB/Wk average - UW & $5.5 \pm 2.8$ & 15.5 & 18.3 & 1.2 & 29 & AD 1665-1945 \\
UB/Wk average - Pta/Grn & $1.4 \pm 5.7$ & 15.4 & 12.1 & 0.8 & 7 & AD 1835-1895 \\
UB/Wk average - NZ & $16.2 \pm 3.5$ & 23.1 & 31.3 & 1.4 & 41 & AD 1335-1745 \\
UB/Wk average - NZ & $39.2 \pm 6.6$ & 23.1 & 26.8 & 1.2 & 12 & AD 1335-1445 \\
\hline
\end{tabular}


Table 3 Combined Southern Hemisphere data from New Zealand, Chile, and South Africa (SHCal02) covering the period AD 955-1955. An error multiplier of 1.2 was applied to the uncertainties from 1665-1955, whereas no additional error term was included prior to this.

\begin{tabular}{crlrrll}
\hline Year AD & \multicolumn{2}{c}{$\Delta^{14} \mathrm{C}(\% \circ)$} & \multicolumn{3}{c}{${ }^{14} \mathrm{C} \mathrm{BP}$} \\
\hline 955 & -25.6 & \pm & 1.7 & 1175 & \pm & 13 \\
965 & -25.8 & \pm & 1.6 & 1167 & \pm & 13 \\
975 & -25.2 & \pm & 1.5 & 1153 & \pm & 12 \\
985 & -24.9 & \pm & 1.5 & 1141 & \pm & 12 \\
995 & -18.3 & \pm & 1.6 & 1077 & \pm & 13 \\
1005 & -20.3 & \pm & 1.6 & 1083 & \pm & 13 \\
1015 & -23.7 & \pm & 1.5 & 1101 & \pm & 12 \\
1025 & -18.1 & \pm & 1.6 & 1045 & \pm & 13 \\
1035 & -15.2 & \pm & 1.6 & 1012 & \pm & 12 \\
1045 & -16.0 & \pm & 1.5 & 1009 & \pm & 12 \\
1055 & -8.8 & \pm & 1.7 & 940 & \pm & 14 \\
1065 & -14.5 & \pm & 1.8 & 977 & \pm & 14 \\
1075 & -12.6 & \pm & 1.8 & 953 & \pm & 14 \\
1085 & -19.5 & \pm & 1.6 & 999 & \pm & 13 \\
1095 & -22.3 & \pm & 1.7 & 1012 & \pm & 14 \\
1105 & -22.1 & \pm & 1.5 & 1001 & \pm & 12 \\
1115 & -21.8 & \pm & 1.6 & 988 & \pm & 13 \\
1125 & -24.9 & \pm & 1.7 & 1004 & \pm & 14 \\
1135 & -24.6 & \pm & 1.5 & 992 & \pm & 12 \\
1145 & -24.7 & \pm & 1.5 & 983 & \pm & 12 \\
1155 & -21.6 & \pm & 1.5 & 948 & \pm & 12 \\
1165 & -19.0 & \pm & 1.5 & 917 & \pm & 12 \\
1175 & -23.0 & \pm & 1.5 & 940 & \pm & 12 \\
1185 & -19.3 & \pm & 1.5 & 900 & \pm & 12 \\
1195 & -20.7 & \pm & 1.7 & 902 & \pm & 14 \\
1205 & -22.3 & \pm & 1.5 & 905 & \pm & 12 \\
1215 & -20.8 & \pm & 1.7 & 883 & \pm & 14 \\
1225 & -17.3 & \pm & 1.6 & 845 & \pm & 13 \\
1235 & -15.4 & \pm & 1.6 & 819 & \pm & 13 \\
1245 & -17.3 & \pm & 1.6 & 825 & \pm & 13 \\
1255 & -20.6 & \pm & 1.5 & 843 & \pm & 12 \\
1265 & -20.4 & \pm & 1.5 & 832 & \pm & 12 \\
1275 & -17.3 & \pm & 1.7 & 796 & \pm & 14 \\
1285 & -13.3 & \pm & 1.7 & 754 & \pm & 14 \\
1295 & -10.6 & \pm & 1.6 & 722 & \pm & 13 \\
1305 & -8.1 & \pm & 1.6 & 693 & \pm & 13 \\
1315 & -9.6 & \pm & 1.4 & 694 & \pm & 11 \\
1325 & -3.9 & \pm & 1.6 & 639 & \pm & 13 \\
1335 & -5.3 & \pm & 1.6 & 641 & \pm & 13 \\
1345 & -7.5 & \pm & 1.5 & 648 & \pm & 12
\end{tabular}


Table 3 Combined Southern Hemisphere data from New Zealand, Chile, and South Africa (SHCal02) covering the period AD 955-1955. An error multiplier of 1.2 was applied to the uncertainties from 1665-1955, whereas no additional error term was included prior to this. (Continued)

\begin{tabular}{lrlllll}
\hline Year AD & \multicolumn{2}{c}{$\Delta^{14} \mathrm{C}(\%)$} & \multicolumn{3}{c}{${ }^{14} \mathrm{C} \mathrm{BP}$} \\
\hline 1355 & -12.7 & \pm & 1.5 & 681 & \pm & 12 \\
1365 & -17.2 & \pm & 1.6 & 708 & \pm & 13 \\
1375 & -19.3 & \pm & 1.6 & 716 & \pm & 13 \\
1385 & -15.3 & \pm & 1.6 & 673 & \pm & 13 \\
1395 & -10.7 & \pm & 1.7 & 626 & \pm & 14 \\
1405 & -7.2 & \pm & 1.2 & 587 & \pm & 9 \\
1415 & -5.5 & \pm & 1.1 & 564 & \pm & 9 \\
1425 & -4.3 & \pm & 1.1 & 545 & \pm & 9 \\
1435 & -3.1 & \pm & 1.1 & 525 & \pm & 9 \\
1445 & -0.9 & \pm & 1.1 & 498 & \pm & 9 \\
1455 & 3.2 & \pm & 1.5 & 455 & \pm & 12 \\
1465 & 7.8 & \pm & 1.5 & 409 & \pm & 12 \\
1475 & 3.9 & \pm & 1.7 & 430 & \pm & 14 \\
1485 & 6.0 & \pm & 1.5 & 404 & \pm & 12 \\
1495 & 4.1 & \pm & 1.6 & 409 & \pm & 13 \\
1505 & 6.9 & \pm & 1.5 & 377 & \pm & 12 \\
1515 & 9.9 & \pm & 1.5 & 343 & \pm & 12 \\
1525 & 8.6 & \pm & 1.5 & 345 & \pm & 12 \\
1535 & 7.7 & \pm & 1.6 & 342 & \pm & 13 \\
1545 & 5.2 & \pm & 1.6 & 352 & \pm & 13 \\
1555 & 2.4 & \pm & 1.6 & 365 & \pm & 13 \\
1565 & 2.8 & \pm & 1.5 & 352 & \pm & 12 \\
1575 & 0.6 & \pm & 1.5 & 359 & \pm & 12 \\
1585 & -1.2 & \pm & 1.6 & 364 & \pm & 13 \\
1595 & -5.4 & \pm & 1.6 & 388 & \pm & 13 \\
1605 & -8.4 & \pm & 1.5 & 403 & \pm & 12 \\
1615 & -7.4 & \pm & 1.5 & 385 & \pm & 12 \\
1625 & -3.8 & \pm & 1.5 & 346 & \pm & 12 \\
1635 & -2.5 & \pm & 1.5 & 327 & \pm & 12 \\
1645 & 0.0 & \pm & 1.5 & 297 & \pm & 12 \\
1655 & 4.9 & \pm & 1.5 & 248 & \pm & 12 \\
1665 & 2.5 & \pm & 1.5 & 257 & \pm & 12 \\
1675 & 7.8 & \pm & 0.9 & 204 & \pm & 7 \\
1685 & 9.7 & \pm & 0.8 & 179 & \pm & 6 \\
1695 & 10.3 & \pm & 0.6 & 165 & \pm & 5 \\
1705 & 10.7 & \pm & 0.5 & 153 & \pm & 4 \\
1715 & 11.4 & \pm & 0.5 & 138 & \pm & 4 \\
1725 & 7.3 & \pm & 1.0 & 161 & \pm & 8 \\
1735 & 0.8 & \pm & 1.2 & 203 & \pm & 10 \\
1745 & -2.5 & \pm & 1.2 & 220 & \pm & 10
\end{tabular}


Table 3 Combined Southern Hemisphere data from New Zealand, Chile, and South Africa (SHCal02) covering the period AD 955-1955. An error multiplier of 1.2 was applied to the uncertainties from 1665-1955, whereas no additional error term was included prior to this. (Continued)

\begin{tabular}{lrlllll}
\hline Year AD & \multicolumn{3}{c}{$\Delta^{14} \mathrm{C}(\% \circ)$} & \multicolumn{3}{c}{${ }^{14} \mathrm{C} \mathrm{BP}$} \\
\hline 1755 & -4.0 & \pm & 1.2 & 222 & \pm & 10 \\
1765 & -5.4 & \pm & 1.2 & 224 & \pm & 10 \\
1775 & -5.3 & \pm & 0.7 & 212 & \pm & 6 \\
1785 & -8.8 & \pm & 0.8 & 232 & \pm & 6 \\
1795 & -10.0 & \pm & 0.8 & 231 & \pm & 6 \\
1805 & -4.2 & \pm & 0.7 & 175 & \pm & 6 \\
1815 & -0.2 & \pm & 0.7 & 134 & \pm & 6 \\
1825 & 0.3 & \pm & 1.2 & 118 & \pm & 9 \\
1835 & -4.6 & \pm & 0.9 & 148 & \pm & 7 \\
1845 & -6.5 & \pm & 0.9 & 154 & \pm & 7 \\
1855 & -7.1 & \pm & 0.7 & 150 & \pm & 6 \\
1865 & -8.2 & \pm & 0.8 & 148 & \pm & 6 \\
1875 & -10.4 & \pm & 0.6 & 156 & \pm & 5 \\
1885 & -9.9 & \pm & 0.7 & 142 & \pm & 6 \\
1895 & -8.4 & \pm & 0.9 & 119 & \pm & 7 \\
1905 & -11.6 & \pm & 0.8 & 137 & \pm & 7 \\
1915 & -12.6 & \pm & 0.9 & 136 & \pm & 8 \\
1925 & -14.8 & \pm & 1.2 & 144 & \pm & 10 \\
1935 & -17.3 & \pm & 1.2 & 155 & \pm & 10 \\
1945 & -20.7 & \pm & 1.2 & 173 & \pm & 10 \\
1955 & -22.1 & \pm & 2.2 & 177 & \pm & 17 \\
\hline
\end{tabular}

\section{CONCLUSIONS}

A comparison between 2 sets of ${ }^{14} \mathrm{C}$ measurements on decadal samples of oak, cedar, and silver pine is presented covering the period AD 955-1845. The results show a periodicity in the offset of about $130 \mathrm{yr}$ with a large change in amplitude centered around AD 1300.

We propose the use of the Southern Hemisphere data presented in Table 3 for calibrating samples from the Southern Hemisphere over the 2nd millennium AD. On the basis of the difference between this and IntCal98, we suggest that the Southern Hemisphere offset value that should be used for calibration prior to $\mathrm{AD} 955$ is $41 \pm 14$. The ${ }^{14} \mathrm{C}$ calibration program CALIB at www.calib.org includes the new Southern Hemisphere data set and provides the option to calibrate Holocene Southern Hemisphere data before AD 955 using the new recommended offset.

While our model study is still in the preliminary stages, it does point out that increased upwelling in the Southern Ocean, caused by either increased deep water formation there or in the North Atlantic, could be responsible for the increased difference in atmospheric ${ }^{14} \mathrm{C}$ around $\mathrm{AD} 1300-1400$. Whatever the cause of the variation in the inter-hemispheric difference, correction of Southern Hemispheric ${ }^{14} \mathrm{C}$ ages with a constant offset could result in an error of up to $50 \mathrm{yr}$. 


\section{ACKNOWLEDGMENTS}

This work was supported by research grants from the Natural Environment Research Council, Grant No GR9/02597 to Prof F G McCormac at Queen's University of Belfast, and by 2 grants to Drs A G Hogg and T F G Higham at the University of Waikato by the New Zealand Foundation for Research Science and Technology, Grant numbers UOW-508 \& UOW-609. Dave Brown, Stephen Hoper, Jim McDonald, Ollie Lavery, Michelle Thompson (QUB), Helen McKinnon, Margaret Rabjohns, and Dr Fiona Petchey (UOW) contributed to the preparation of wood samples and maintenance of counter quality controls.

\section{REFERENCES}

Braziunas TF, Fung IY, Stuiver M. 1995. The preindustrial atmospheric ${ }^{14} \mathrm{CO}_{2}$ latitudinal gradient as related to exchanges among atmospheric, oceanic, and terrestrial reservoirs. Global Biogeochemical Cycles 9(4): 565-84.

Dettinger MD, Ghil M, Strong CM, Keppenne CL. 1995. Interannual and interdecadal variability in United States surface-air temperatures 1910-87. Climatic Change 31:35-66.

Hogg AG, McCormac FG, Higham TFG, Reimer PG, Baillie MGL, Palmer JG. 2002. High-precision ${ }^{14} \mathrm{C}$ measurements of contemporaneous tree-ring dated wood from the British Isles and New Zealand: AD 1850-950. Radiocarbon. This issue.

Lerman JC, Mook WG, Vogel JC. $1970 .{ }^{14} \mathrm{C}$ in tree-rings from different localities. In: Olsson IU, editor. Radiocarbon variations and absolute chronology: proceedings of the Twelfth Nobel Symposium held at the Institute of Physics at Uppsala University. Stockholm: Almquist and Wiksell. 652 p.

Levin I, Kromer B, Wagenbach D, Munnich KO. 1987. Tellus 39B:89-95.

McCormac FG, Hogg AG, Higham TFG, Lynch-Stieglitz J, Broecker WS, Baillie MGL, Palmer J, Xiong L, Pilcher JR, Brown D, Hoper ST. 1998. Variations of radiocarbon in tree rings: Southern Hemisphere offset preliminary results. Radiocarbon 40(3):1153-59.

McCormac FG, Baillie MGL, Pilcher JR, Kalin RM. 1995. Location-dependent differences in the ${ }^{14} \mathrm{C}$ content of wood. Radiocarbon 37(2):395-407.
Mitchell et al. 1966. World Meteorological Organization. Technical Note $79.79 \mathrm{p}$.

Reimer PJ, McCormac FG, Reimer RW, Braziunas TF, Hogg AG, Higham TFG. Forthcoming. Interhemispheric variation in the response to solar forcing over the past 1000 yr. Science.

Sparks RJ, Melhuish H, McKee JWA, Ogden J, Palmer JG, Molloy BPJ. 1995. ${ }^{14} \mathrm{C}$ calibration in the Southern Hemisphere and the date of the last Taupo eruption: evidence from tree-ring sequences. Radiocarbon 37(2): 155-63.

Stuiver M, Braziunas TF. 1998. Anthropogenic and solar components of hemispheric ${ }^{14} \mathrm{C}$. Geophysical Research Letters 25:329-32.

Stuiver M, Pearson GW. 1986. High-precision calibration of the Radiocarbon time scale, AD 1950-500 BC. Radiocarbon 28(2B):805-38.

Stuiver M, Reimer PJ, Bard E, Beck JW, Burr GS, Hughen KA, Kromer B, McCormac FG, van der Plicht J, Spurk M. 1998. INTCAL98 radiocarbon age calibration, 24,000-0 cal BP. Radiocarbon 40(3):104183.

Vautard R, Yiou P, Ghil M. 1992. Singular-spectrum analysis-a toolkit for short, noisy chaotic signals. Physica D 58:95-126.

Vogel JC, Fuls A, Visser E, Becker B. 1993. Pretoria calibration curve for short-lived samples 1930-3350 BC. In: Stuiver M, Kra RS, editors. Calibration 1993 issue. Radiocarbon 35(1):73-85. 